Article

\title{
A Cross-Cultural Comparison of the Link between Modernization, Anthropomorphism and Attitude to Wildlife
}

\author{
Jose Luis Gomez-Melara ${ }^{1, *(1)}$, Rufino Acosta-Naranjo ${ }^{1}$, Patricia Izar ${ }^{2}$, Shahrul Anuar Mohd Sah ${ }^{3}$, \\ Jordi Pladevall ${ }^{4}$, Risma Illa Maulany ${ }^{5}$, Putu Oka Ngakan ${ }^{5}$, Bonaventura Majolo ${ }^{6}{ }^{(}$, Teresa Romero ${ }^{7}$ \\ and Federica Amici $8,9, * \mathbb{B}$
}

check for

updates

Citation: Gomez-Melara, J.L.; Acosta-Naranjo, R.; Izar, P.; Sah, S.A.M.; Pladevall, J.; Maulany, R.I.; Ngakan, P.O.; Majolo, B.; Romero, T.; Amici, F. A Cross-Cultural Comparison of the Link between Modernization, Anthropomorphism and Attitude to Wildlife. Sustainability 2021, 13, 13095. https://doi.org/ $10.3390 /$ su132313095

Academic Editor: Reuven Yosef

Received: 24 September 2021

Accepted: 19 November 2021

Published: 26 November 2021

Publisher's Note: MDPI stays neutral with regard to jurisdictional claims in published maps and institutional affiliations.

Copyright: (c) 2021 by the authors. Licensee MDPI, Basel, Switzerland. This article is an open access article distributed under the terms and conditions of the Creative Commons Attribution (CC BY) license (https:// creativecommons.org/licenses/by/ $4.0 /)$.
1 Department of Social Anthropology, University of Seville, 41004 Seville, Spain; racosta@us.es

2 Department of Experimental Psychology, University of São Paulo, São Paulo 05508, Brazil; patrizar@usp.b

3 School of Biological Sciences, Universiti Sains Malaysia, 1112, Persiaran Sains, Gelugor 11800, Malaysia; anuarusm@gmail.com

4 University of Girona Foundation: Innovation and Training, University of Girona, 17004 Girona, Spain; plaaade@gmail.com

5 Forestry Department, Hasanuddin University, Makassar 90245, Indonesia; riezzy2000@yahoo.com.au (R.I.M.); ngakanpo@gmail.com (P.O.N.)

6 School of Psychology, University of Lincoln, Lincoln LN6 7TS, UK; bmajolo@lincoln.ac.uk

7 School of Life Sciences, University of Lincoln, Lincoln LN6 7TS, UK; tromero@lincoln.ac.uk

8 Institute of Biology, Faculty of Life Science, University of Leipzig, 04109 Leipzig, Germany

9 Department of Human Behaviour, Ecology and Culture, Max-Planck Institute for Evolutionary Anthropology, 04103 Leipzig, Germany

* Correspondence: jgmelara@us.es (J.L.G.-M.); amici@eva.mpg.de (F.A.)

\begin{abstract}
Anthropogenic pressure has significantly increased in the last decades, often enhancing conflicts at the human-wildlife interface. Therefore, understanding peoples' value orientations, attitudes and behavioural intentions towards wildlife is a crucial endeavour to reduce the occurrence of conflicts between humans and wildlife. Previous research in the USA has shown a consistent link between modernization and increased anthropomorphism (i.e., the tendency to attribute human mental or physical characteristics to other entities), leading to positive changes in value orientations, attitudes, and behavioural intentions towards wildlife. In this paper, we aimed to address whether this link is also present in other cultures, by testing participants $(\mathrm{N}=741)$ in five different countries (Brazil, Indonesia, Malaysia, Mexico, and Spain). Our study shows that while the positive link between anthropomorphism, positive attitudes and behavioural intentions towards wildlife is universal, the link between modernization and anthropomorphism is culturally mediated. In some countries (Indonesia, Malaysia, Spain), modernization increased anthropomorphism, while in others modernization predicted no differences (Brazil) or even a decrease in anthropomorphism (Mexico), ultimately deteriorating individuals' attitude and behavioural intentions towards wildlife. These results call for caution when generalizing findings from western industrialized countries to inform conservation policies worldwide.
\end{abstract}

Keywords: modernization; anthropomorphism; domination; mutualism; attitude; wildlife

\section{Introduction}

Anthropogenic pressure on the environment (i.e., any human activity that may have a direct or indirect impact on ecosystems, such as hunting, logging or pollution derived from industry) has significantly increased in the last decades, often enhancing conflicts at the human-wildlife interface, with consequences for human safety, disease transmission and biodiversity loss, just to name a few (e.g., [1-3]). Therefore, decreasing the occurrence of these conflicts appears a major endeavour for politics and science [4,5]. One way to reduce the occurrence and/or intensity of conflicts between humans and wildlife is to improve peoples' attitudes toward wildlife, for instance through education programs that 
increase environmental knowledge [6,7]. However, other factors might also affect human attitude toward wildlife. Domination orientations, for instance, emphasize the existence of a hierarchical division between human and non-human animals, and are thought to lead to a utilitarian attitude to wildlife, in which the environment is mainly managed to increase human welfare (e.g., [8,9]). Males and older people, for instance, are more likely to have domination orientations than females and younger individuals [10]. In contrast, mutualism orientations stress how humans and other animals are part of the same socio-ecological world, leading to positive feelings, social attachment and a more positive attitude toward wildlife [11-13]. Mutualism orientations, for instance, are thought to be higher in females and in individuals living in more urban areas, as compared with males and individuals from more rural areas [10].

Recently, researchers have proposed that an increase in modernization, a broad term including economic wealth, urbanization and formal education, might have caused a change in the relationship between humans and wildlife, by altering human orientations (i.e., domination or mutualism), attitude and behaviours toward wildlife (see [9,12-14]). In particular, modernization has led people in post-industrial countries to experience increased loneliness and social isolation [15,16], but also less frequent encounters with wildlife and thus a more benign and less conflictive association with animals (e.g., through pet ownership; see [12,17]). According to this hypothesis, the tendency to attribute human mental or physical characteristics to other entities (i.e., anthropomorphism), further fostered by an affective relationship with pets, would have enhanced human empathy and perception of similarity with other species (see $[18,19])$. In turn, this would have led humans to switch from domination to mutualism orientations, and to more positive attitudes and behaviours toward animals [9,11,12,14,15,19-21].

In the USA, individuals scoring higher in urbanization, income and education were more likely to attribute free will, consciousness or ability to experience emotions to wildlife, and to describe humans and other species as being part of the same family, sharing the same rights [19]. These participants also had a more positive attitude toward wildlife in case of conflict, more likely rejecting the idea of using animals for their own benefit [19]. These results are in line with other studies showing that humans are more willing to invest in the conservation of species that are phylogenetically closer to humans and thus more likely to be anthropomorphized [22-26]. In other countries, the link between anthropomorphism, mutualism and/or better attitude toward animals is also relatively well established (see [27]). Anthropomorphism, for instance, predicted students' intentions to become vegetarian and vegan in Spain [28], worse attitudes toward meat consumption in the USA [29], and a better attitude toward several species in the UK [30]. Anthropomorphism also correlated to mutualism and positive attitudes toward animals in Romania [31], and to positive attitudes toward nature in Singapore [32,33] and Hong Kong [34]. These effects are evident despite important inter-individual differences in anthropomorphism, which are linked to individuals' gender [35,36], age [37] and emotional attachment to pets [38]. In Germany, in contrast, anthropomorphism had little effect on attitude toward fishing, although mutualism was linked to a negative perception of these practices [39]. Besides the work conducted in the USA, however, little is known about the link between modernization and anthropomorphism. In particular, it is not clear whether higher modernization generally predicts an increase in anthropomorphism across countries, with positive changes in orientations and attitudes.

In this study, we aimed to analyse the complex relationship between modernization, anthropomorphism, mutualism/domination orientations and attitude towards wildlife, by using a cross-cultural approach. We built on the work by Manfredo et al. [19], adapting it in the following ways. First, we conducted research in five countries in three continents (i.e., Brazil, Indonesia, Malaysia, Mexico, and Spain), using the same methodology to allow a reliable cross-cultural comparison. Second, we assessed several aspects of modernization beyond the ones used by Manfredo at al. [19] (i.e., urbanization, income and formal education), also including questions about participants' use of technologies, sociality and 
relationship with animals (see Methods and Appendix B for more details). Finally, we included a new set of questions to assess participants' attitude toward wildlife, and directly tested its link to anthropomorphism, mutualism and domination.

We predicted that modernization would be linked to an increase in anthropomorphism, but that this effect would be culturally mediated, varying across countries (Prediction 1; Table 1). We further predicted that higher anthropomorphism would be linked to lower domination and higher mutualism (independently of participants' country; Prediction 2; Table 1). Finally, we predicted that participants with lower domination and higher mutualism would show more positive attitudes towards wildlife, independently of their country (Prediction 3; Table 1).

Table 1. Predictions of our study, models used to test them, and whether they were confirmed.

\begin{tabular}{ccc}
\hline Prediction & Model & Confirmed? \\
\hline $\begin{array}{c}\text { 1. Modernization is linked to higher anthropomorphism, } \\
\text { but this effect is culturally mediated }\end{array}$ & M1 & Yes \\
\hline $\begin{array}{c}\text { 2. Anthropomorphism predicts lower domination and } \\
\text { higher mutualism orientation }\end{array}$ & M2a-M2b & Yes \\
\hline $\begin{array}{c}\text { 3. Higher mutualism and lower domination orientation } \\
\text { are linked to a more positive attitude towards wildlife }\end{array}$ & M3 & Yes \\
\hline
\end{tabular}

\section{Materials and Methods}

\subsection{Ethics}

Participation was voluntary and completely anonymous. Informed consent was obtained from subjects before testing started. Participants were informed about the purpose of the study and were able to withdraw their participation at any time.

\subsection{Participants}

We recruited 741 participants across five countries (Brazil, $\mathrm{N}=156$, Indonesia, $\mathrm{N}=248$, Malaysia, $\mathrm{N}=118$, Mexico, $\mathrm{N}=111$, Spain, $\mathrm{N}=108$ ), for details see Appendix A. We started testing participants in Indonesia $(\mathrm{N}=248)$ and Spain $(\mathrm{N}=19)$ with a printed version of the questionnaire that participants filled in person. However, soon after we started data collection in Spain we had to stop direct interactions with people due to the outbreak of the COVID-19 global pandemic. We therefore switched to an online version of the questionnaire using the ethnographic software Ethnoap. Online recruitment proved to be difficult, but our sample sizes are still in line with other studies on cross-cultural variation in attitude/behaviour to wildlife (e.g., $[28,39,40]$ ). Comparing the two Spanish groups (which were the only countries in which we tested participants both online and in person) revealed no significant differences in their responses (Table 2: for all six comparisons, $p>0.05)$, and therefore we included all participants in the subsequent analyses.

Table 2. Mean ( $\pm \mathrm{SD}$ ) levels of anthropomorphism, domination, mutualism, attitudes, and idioallocentric tendencies in Spain, where participants were tested with different modalities, separately for each modality (i.e., online and paper).

\begin{tabular}{cccccc}
\hline Modality & Anthrop. & Domin. & Mutual. & Attitudes & Idio-Alloc. \\
\hline Online & $0.72 \pm 0.13$ & $0.48 \pm 0.15$ & $0.73 \pm 0.14$ & $0.37 \pm 0.14$ & $0.55 \pm 0.08$ \\
\hline Paper & $0.72 \pm 0.09$ & $0.54 \pm 0.15$ & $0.70 \pm 0.13$ & $0.37 \pm 0.14$ & $0.51 \pm 0.09$ \\
\hline
\end{tabular}

Participants identified themselves as females $(\mathrm{N}=394$, mean age $\pm \mathrm{SD}=28.22 \pm 13.34$, range $=17-78)$ or males $(\mathrm{N}=240$, mean age $\pm \mathrm{SD}=29.57 \pm 13.96$, range $=17-79)$. For more demographic information about the participants, please see Appendix A and B. In Indonesia, participants were opportunistically recruited in the Bira Bonto Bahari beach road area $(\mathrm{N}=100)$, and at the Hasanuddin University by the 6th author $(\mathrm{N}=145)$. In 
Spain, participants were recruited by the 1st author (i.e., in person: by distributing the printed version before the beginning of lectures at the University of Seville, Spain; online: by distributing the Ethnoap link through the online-class platform before the beginning of lectures). In Malaysia, participants were recruited by the 4 th author, and in Brazil and Mexico by the 3rd and 5th authors, respectively, through social media, in both urban and rural communities (i.e., specifically advertising the questionnaire to acquaintances living in both kinds of communities). Although we aimed to recruit a sample representative of the general population, our final sample was biased towards younger individuals with higher formal education (see Appendix A and B for details).

\subsection{Questionnaires}

We used the questionnaire designed and validated by Manfredo et al. [19] to measure anthropomorphism, domination and mutualism orientations. First, we assessed participants' anthropomorphism with 5 statements (e.g., "Animals have free will") that could be rated on a 5-point agreement scale (from "strongly disagree" to "strongly agree"). These measures were combined into an individual modernization score between 0 and 1 (i.e., minimum to maximum level of anthropomorphism; see Appendix B). Second, we assessed participants' domination orientation with 10 statements (e.g., "Humans should manage fish and wildlife populations so that human benefit") rated on a 5-point agreement scale. Similarly, we assessed participants' mutualism orientation, by using 10 statements (e.g., "Hunting is cruel and inhumane to the animals") rated on a 5-point agreement scale. These measures were combined into a domination score and a mutualism score, both between 0 and 1 (i.e., minimum to maximum domination and mutualism levels; see Appendix B). Internal consistency for these three measures was acceptable/good, with Cronbach's alpha ranging from 0.72 for anthropomorphism to 0.85 for mutualism.

We further assessed other possible factors that might be linked to participants' anthropomorphism, mutualism and domination orientations. First, we assessed participants' attitude towards wildlife with 4 questions rated on a 5-point agreement scale (e.g., "Animals are a pest messing up the place where I live"; see Karimullah et al., in review). Responses were then combined into a single score from 0 to 1 (i.e., most negative to most positive attitude towards wildlife). Second, we measured different aspects of participants' degree of modernization, including their (i) individual income, (ii) level of formal education, (iii) modern experience with animals (e.g., "Have you ever visited a zoo, a natural reserve or a similar place?"), and (iv) access to technologies (e.g., use of social media). Please note that the concept of "modernity" (especially in relation to experience with animals) has a neutral connotation, and is simply used to refer to all those human-animal interactions that are more likely to occur in "modern", i.e., more urbanized contexts, where natural encounters with wild animals are less likely. Third, we assessed pet-ownership dynamics (i.e., ownership and affection to pets) with a score ranging from 0 to 1 (i.e., lowest to highest affection to pets), based on responses to 3 yes-or-no questions (e.g., "Do you own any animal?"). Fourth, we assessed participants' sociality (i.e., frequency of social interactions) based on 9 open questions measuring different aspects of sociality (e.g., "How many times do you hang out in an average week?"). The final score ranged from 0 to 9 (i.e., lowest to highest sociality). Fifth, we assessed participants' idiocentric and allocentric tendencies using a validated questionnaire with 16 statements (as in [41,42]), like "I would rather depend on myself than others", rated on a 5-point agreement scale. Finally, we collected demographic information with open questions on participants' age, gender and dietary preferences (i.e., being vegetarian or vegan). For more details on how each score was calculated, please see Appendix B.

\subsection{Data Analysis}

We used linear mixed models and generalized linear mixed models [43-45] with the glmmTMB package (version 1.0.16 [45]) in Ref [44]. We ran four pairs of full-null models. In Model 1 (M1), we aimed to test whether modernization predicted higher anthropomor- 
phism, and whether an individual's country mediated this relationship (see [19]). The anthropomorphism index was our dependent variable, which varied between 0 and 1 and was modelled with a beta distribution. As test predictors in M1, we included the two-way interaction of modernity with country. Moreover, we included as test predictors the idio-allocentric, sociality and pet-ownership indexes, and whether participants' had a vegetarian/vegan diet (as binomial predictor). Participants' age and gender were included as controls.

In Models 2a and 2b, we aimed to test whether higher anthropomorphism predicted lower domination (M2a) and higher mutualism (M2b), and whether this held true across countries. Our dependent variables were domination (in M2a) and mutualism (in M2b), which both varied between 0 and 1 and were thus modelled with a beta distribution. In both models, we included the two-way interaction of anthropomorphism with country as test predictors, and as controls all the other controls and predictors that had been included in M1 (i.e., modernity, idio-allocentric, sociality and pet-ownership indexes, vegetarian/vegan diet, participants' age and gender).

In Model 3 (M3), we aimed to test whether higher mutualism and lower domination predicted a more positive attitude toward wildlife, and whether this was true across countries. Our dependent variable was attitude toward wildlife, which also varied between 0 and 1 and was modelled with a beta distribution. As test predictors we included the two-way interaction of domination with country, as well as the two-way interactions of mutualism with country. As controls we included, as above, all the controls and predictors that had been included in the previous models (i.e., anthropomorphism, modernity, idioallocentric, sociality and pet-ownership indexes, vegetarian/vegan diet, participants' age and gender).

After removing missing data, we had a total of 574 data points for M1, M2a and M2b, and 571 for M3. Before running the models, we $z$-transformed all continuous predictors to facilitate model convergence and interpretation of model coefficients. Dependent variables including $0 \mathrm{~s}$ and/or $1 \mathrm{~s}$ were previously transformed as suggested by Smithson and Verkuilen [46]. We used likelihood ratio tests to compare full models containing all predictors with null models containing only control predictors and random factors. When full models significantly differed from null models, likelihood ratio tests were conducted to obtain the $p$ values for each test predictor via single-term deletion using the $\mathrm{R}$ function drop. When models included two-way interactions, the main terms were also included, and if the interaction was not significant, the model was re-run including only the main terms (e.g., M2a). Post-hoc comparisons were then conducted using Tukey tests for significant categorical predictors or interpreting the slope for continuous predictors. We added significant test predictors of the former full models (e.g., M1) as controls in the following models (e.g., M2), to account for the fact that the same measures could be used as dependent or independent variables in different models (e.g., we tested if anthropomorphism was predicted by modernization, but we also tested whether anthropomorphism predicted domination). If modernization predicted anthropomorphism in M1, for instance, in M2 we tested whether anthropomorphism predicted domination by also including modernization as a control, so that the effect of anthropomorphism on domination could be assessed while controlling for modernization. We detected no overdispersion or convergence issues in any of the models presented. To rule out collinearity, we determined the VIFs, which were acceptable (maximum VIFs across all models $=4.45$ ).

\section{Results}

In M1 we tested whether modernization predicted higher anthropomorphism, and whether one's country mediated this relationship (see Figure 1). The full and null models significantly differed (GLMM: $\chi 2=107.81, \mathrm{df}=13, p<0.001$; Table 3), showing a significant effect of the two-way interaction (modernity-country: $p=0.046$ ). In particular, modernization predicted an increase in anthropomorphism in all countries, except for Latin American countries (i.e., Brazil, where it had no effect on anthropomorphism, and Mexico, 
where higher modernization was linked to lower anthropomorphism). Moreover, modern pet-ownership dynamics $(p=0.006)$ and having a vegetarian/vegan diet $(p=0.012)$ were linked to higher anthropomorphism.

In M2a and M2b we tested whether anthropomorphism predicted lower domination (M2a) and higher mutualism (M2b), and whether this held true across countries. In M2a, the full and null models significantly differed (GLMM: $\chi 2=150.54, \mathrm{df}=9, p<0.001$; Table 3). Domination was higher when anthropomorphism was lower $(p<0.001)$, and it differed across countries $(p<0.001)$, being generally higher in Asian countries (i.e., Malaysia and especially Indonesia), intermediate in Spain and lowest in South America (i.e., Mexico and especially Brazil). In M2b the full and null models were significantly different (GLMM: $\chi 2=248.49, \mathrm{df}=9, p<0.001$; Table 3$)$, with the two-way interaction of country and anthropomorphism significantly predicting mutualism $(p<0.001)$. In particular, higher anthropomorphism predicted higher mutualism across all countries, especially in Malaysia (see Figure 2).

In M3 we tested whether higher mutualism and lower domination predicted a more positive attitude toward wildlife, and whether this was true across countries. The full and null models significantly differed (GLMM: $\chi 2=206.77, \mathrm{df}=14, p<0.001$; Table 3), showing a significant effect of both two-way interactions (domination-country: $p=0.043$, see Figure 3; mutualism-country: $p<0.001$, see Figure 4). In particular, higher domination predicted worse attitude in all countries, except in Malaysia, while higher mutualism predicted better attitude in all countries, especially in Malaysia.

Table 3. Results of the models run, including estimates, standard errors (SE), confidence intervals (CIs), likelihood ratio tests (LRT), degrees of freedom (df), and $p$ values for each test and control predictor. Control predictors are in italics. All continuous test predictors and controls were $z$-transformed prior to analysis. The asterisks denote significant $p$ values for the test predictors. All models had a beta distribution.

\begin{tabular}{|c|c|c|c|c|c|c|c|}
\hline Model & Estimate & SE & $2.5 \% \mathrm{CI}$ & $97.5 \% \mathrm{CI}$ & LRT & df & $p$ \\
\hline \multicolumn{8}{|c|}{ Model 1: Anthropomorphism } \\
\hline Intercept & 1.53 & 0.12 & 1.30 & 1.77 & - & - & - \\
\hline Country (Indonesia) * Modernization & 0.07 & 0.14 & -0.21 & 0.34 & \multirow{4}{*}{9.71} & \multirow{4}{*}{4} & \multirow{4}{*}{$0.046^{*}$} \\
\hline Country (Malaysia) * Modernization & 0.20 & 0.19 & -0.17 & 0.58 & & & \\
\hline Country (Mexico)* Modernization & -0.19 & 0.16 & -0.50 & 0.12 & & & \\
\hline Country (Spain) * Modernization & 0.26 & 0.18 & -0.09 & 0.61 & & & \\
\hline Country (Indonesia) & -0.56 & 0.15 & -0.85 & -0.27 & \multirow{4}{*}{-} & \multirow{4}{*}{-} & \multirow{4}{*}{-} \\
\hline Country (Malaysia) & 0.25 & 0.17 & -0.08 & 0.58 & & & \\
\hline Country (Mexico) & -0.12 & 0.16 & -0.44 & 0.19 & & & \\
\hline Country (Spain) & -0.68 & 0.16 & -0.98 & -0.37 & & & \\
\hline Modernization & 0.01 & 0.13 & -0.24 & 0.26 & - & - & - \\
\hline Idio-allocentrism & -0.02 & 0.04 & -0.09 & 0.06 & 0.20 & 1 & 0.655 \\
\hline Pet-ownership & 0.10 & 0.04 & 0.03 & 0.18 & 7.50 & 1 & 0.006 \\
\hline Sociality & 0.02 & 0.04 & -0.06 & 0.10 & 0.19 & 1 & 0.661 \\
\hline Vegetarianism & 0.23 & 0.09 & 0.05 & 0.42 & 6.28 & 1 & 0.012 \\
\hline Gender & 0.01 & 0.08 & -0.13 & 0.16 & 0.03 & 1 & 0.857 \\
\hline Age & -0.06 & 0.05 & -0.15 & 0.04 & 1.40 & 1 & 0.237 \\
\hline
\end{tabular}


Table 3. Cont.

\begin{tabular}{|c|c|c|c|c|c|c|c|}
\hline Model & Estimate & SE & $2.5 \% \mathrm{CI}$ & $97.5 \% \mathrm{CI}$ & LRT & df & $p$ \\
\hline \multicolumn{8}{|c|}{ Model 2a: Domination } \\
\hline Intercept & -0.65 & 0.06 & -0.77 & -0.53 & - & - & - \\
\hline Country (Indonesia) & 0.83 & 0.08 & 0.68 & 0.98 & \multirow{4}{*}{116.60} & \multirow{4}{*}{4} & \multirow{4}{*}{$<0.001$ * } \\
\hline Country (Malaysia) & 0.70 & 0.08 & 0.54 & 0.86 & & & \\
\hline Country (Mexico) & 0.33 & 0.07 & 0.19 & 0.47 & & & \\
\hline Country (Spain) & 0.55 & 0.08 & 0.39 & 0.71 & & & \\
\hline Anthropomorphism & -0.09 & 0.02 & -0.13 & -0.06 & 22.45 & 1 & $<0.001^{*}$ \\
\hline Modernization & -0.03 & 0.02 & -0.07 & 0.02 & 1.41 & 1 & 0.234 \\
\hline Idio-allocentrism & 0.04 & 0.02 & 0.00 & 0.08 & 3.88 & 1 & 0.049 \\
\hline Pet-ownership & -0.02 & 0.02 & -0.06 & 0.02 & 1.20 & 1 & 0.274 \\
\hline Sociality & 0.02 & 0.02 & -0.02 & 0.07 & 1.05 & 1 & 0.306 \\
\hline Vegetarianism & -0.17 & 0.05 & -0.27 & -0.08 & 12.84 & 1 & $<0.001$ \\
\hline Gender & 0.22 & 0.04 & 0.14 & 0.30 & 30.50 & 1 & $<0.001$ \\
\hline Age & 0.03 & 0.02 & -0.01 & 0.08 & 1.87 & 1 & 0.172 \\
\hline \multicolumn{8}{|c|}{ Model 2b: Mutualism } \\
\hline Intercept & 1.56 & 0.09 & 1.38 & 1.73 & - & - & - \\
\hline $\begin{array}{l}\text { Country (Indonesia)* } \\
\text { Anthropomorphism }\end{array}$ & -0.07 & 0.08 & -0.22 & 0.08 & \multirow{4}{*}{28.99} & \multirow{4}{*}{4} & \multirow{4}{*}{$<0.001$ * } \\
\hline $\begin{array}{l}\text { Country (Malaysia)* } \\
\text { Anthropomorphism }\end{array}$ & 0.32 & 0.10 & 0.13 & 0.51 & & & \\
\hline $\begin{array}{l}\text { Country (Mexico)* } \\
\text { Anthropomorphism }\end{array}$ & 0.05 & 0.08 & -0.11 & 0.21 & & & \\
\hline Country (Spain) * Anthropomorphism & -0.21 & 0.09 & -0.39 & -0.04 & & & \\
\hline Country (Indonesia) & -0.53 & 0.11 & -0.74 & -0.32 & - & - & - \\
\hline Country (Malaysia) & -0.15 & 0.13 & -0.41 & 0.11 & - & - & - \\
\hline Country (Mexico) & 0.11 & 0.11 & -0.11 & 0.32 & - & - & - \\
\hline Country (Spain) & -0.56 & 0.12 & -0.79 & -0.33 & - & - & - \\
\hline Anthropomorphism & 0.35 & 0.06 & 0.24 & 0.46 & - & - & - \\
\hline Modernization & 0.09 & 0.03 & 0.03 & 0.15 & 7.73 & 1 & $0.005 *$ \\
\hline Idio-allocentrism & -0.06 & 0.03 & -0.11 & -0.01 & 4.78 & 1 & 0.029 \\
\hline Pet-ownership & 0.13 & 0.03 & 0.07 & 0.18 & 21.07 & 1 & $<0.001$ \\
\hline Sociality & -0.01 & 0.03 & -0.07 & 0.05 & 0.15 & 1 & 0.700 \\
\hline Vegetarianism & 0.11 & 0.07 & -0.03 & 0.24 & 2.43 & 1 & 0.119 \\
\hline Gender & -0.13 & 0.06 & -0.24 & -0.03 & 5.78 & 1 & 0.016 \\
\hline Age & -0.03 & 0.03 & -0.10 & 0.04 & 0.76 & 1 & 0.383 \\
\hline \multicolumn{8}{|c|}{ Model 3: Attitude } \\
\hline Intercept & -0.55 & 0.10 & -0.74 & -0.36 & - & - & - \\
\hline Country (Indonesia) ${ }^{*}$ Domination & 0.03 & 0.10 & -0.17 & 0.22 & \multirow{4}{*}{9.84} & \multirow{4}{*}{4} & \multirow{4}{*}{0.043 * } \\
\hline Country (Malaysia) * Domination & -0.29 & 0.13 & -0.53 & -0.04 & & & \\
\hline Country (Mexico) * Domination & -0.14 & 0.12 & -0.37 & 0.08 & & & \\
\hline Country $($ Spain $) *$ Domination & -0.05 & 0.10 & -0.26 & 0.15 & & & \\
\hline
\end{tabular}


Table 3. Cont.

\begin{tabular}{|c|c|c|c|c|c|c|c|}
\hline Model & Estimate & SE & $2.5 \% \mathrm{CI}$ & $97.5 \% \mathrm{CI}$ & LRT & df & $p$ \\
\hline Country (Indonesia) * Mutualism & -0.15 & 0.09 & -0.32 & 0.02 & \multirow{4}{*}{24.85} & \multirow{4}{*}{4} & \multirow{4}{*}{$<0.001$ * } \\
\hline Country (Malaysia)* Mutualism & -0.47 & 0.11 & -0.68 & -0.25 & & & \\
\hline Country (Mexico) * Mutualism & -0.13 & 0.10 & -0.34 & 0.07 & & & \\
\hline Country (Spain) * Mutualism & 0.00 & 0.10 & -0.18 & 0.19 & & & \\
\hline Country (Indonesia) & 0.55 & 0.12 & 0.32 & 0.77 & - & - & - \\
\hline Country (Malaysia) & 0.96 & 0.13 & 0.70 & 1.21 & - & - & - \\
\hline Country (Mexico) & 0.23 & 0.12 & 0.00 & 0.45 & - & - & - \\
\hline Country (Spain) & 0.05 & 0.12 & -0.18 & 0.28 & - & - & - \\
\hline Domination & 0.20 & 0.08 & 0.04 & 0.36 & - & - & - \\
\hline Mutualism & -0.02 & 0.07 & -0.17 & 0.12 & - & - & - \\
\hline Anthropomorphism & -0.02 & 0.03 & -0.07 & 0.04 & 0.40 & 1 & 0.527 \\
\hline Modernization & -0.05 & 0.03 & -0.10 & 0.01 & 2.55 & 1 & 0.110 \\
\hline Idio-allocentrism & 0.03 & 0.02 & -0.02 & 0.08 & 1.51 & 1 & 0.218 \\
\hline Pet-ownership & -0.04 & 0.03 & -0.09 & 0.01 & 2.18 & 1 & 0.140 \\
\hline Sociality & 0.04 & 0.03 & -0.01 & 0.10 & 2.15 & 1 & 0.143 \\
\hline Vegetarianism & -0.05 & 0.06 & -0.17 & 0.07 & 0.65 & 1 & 0.419 \\
\hline Gender & 0.05 & 0.05 & -0.05 & 0.15 & 1.08 & 1 & 0.299 \\
\hline Age & 0.10 & 0.03 & 0.03 & 0.16 & 9.12 & 1 & 0.003 \\
\hline
\end{tabular}

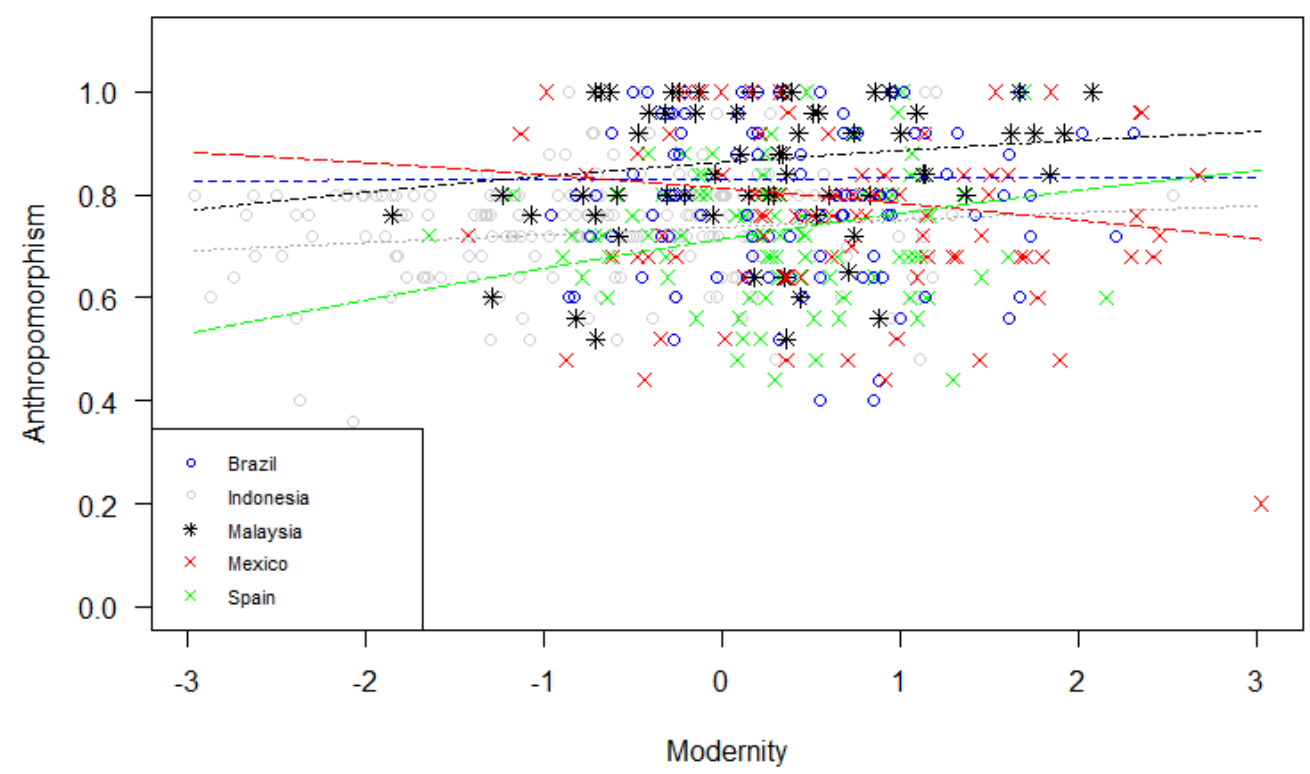

Figure 1. Mean level of participants' anthropomorphism, as a function of their country and modernity. Circles, crosses, and asterisks represent participants. The dashed lines depict the model (as in model 1, but separately for each country), which has been back-transformed from the log-odds ratio scale and contains standardized controls. 


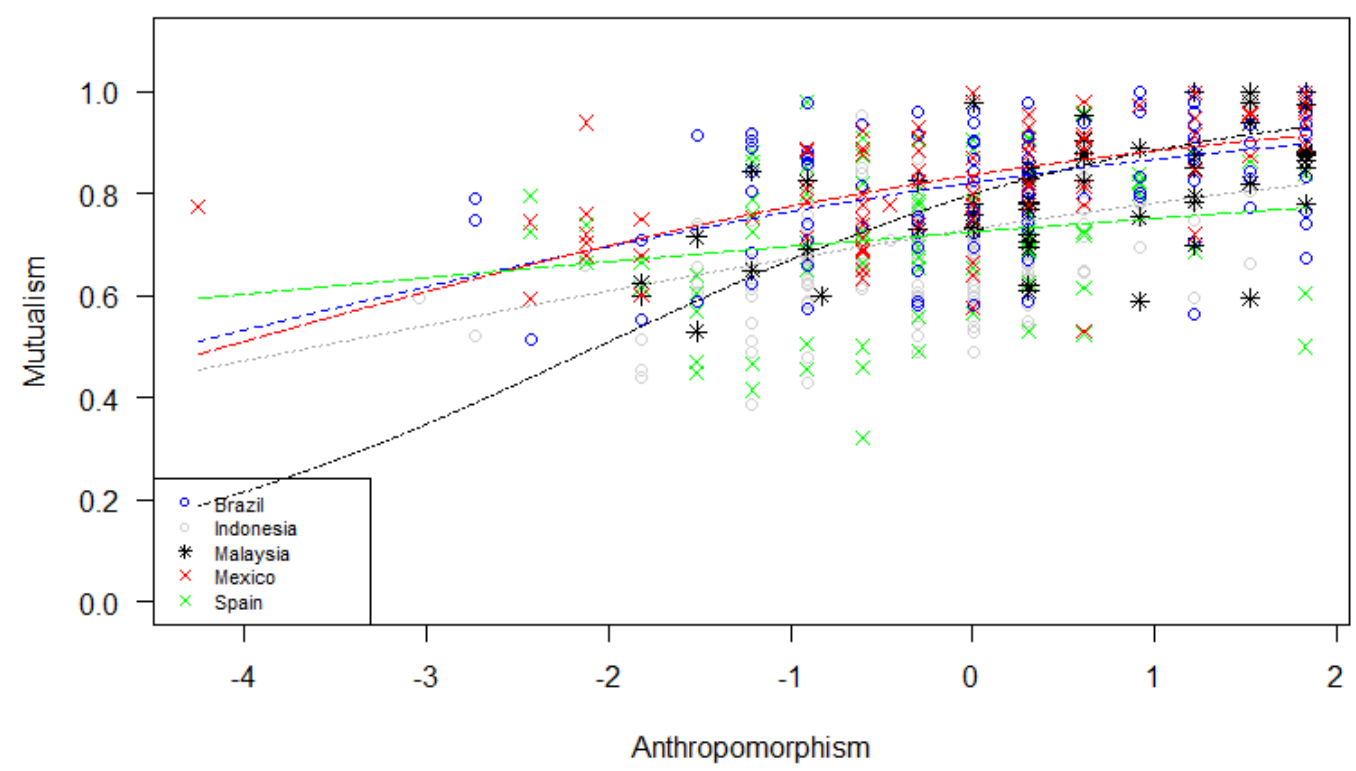

Figure 2. Mean level of participants' mutualism orientations, as a function of their country and anthropomorphism. Circles, crosses, and asterisks represent participants and are jittered to avoid overlap. The dashed lines depict the model (as in model $2 b$, but separately for each country), which has been back-transformed from the log-odds ratio scale and contains standardized controls.

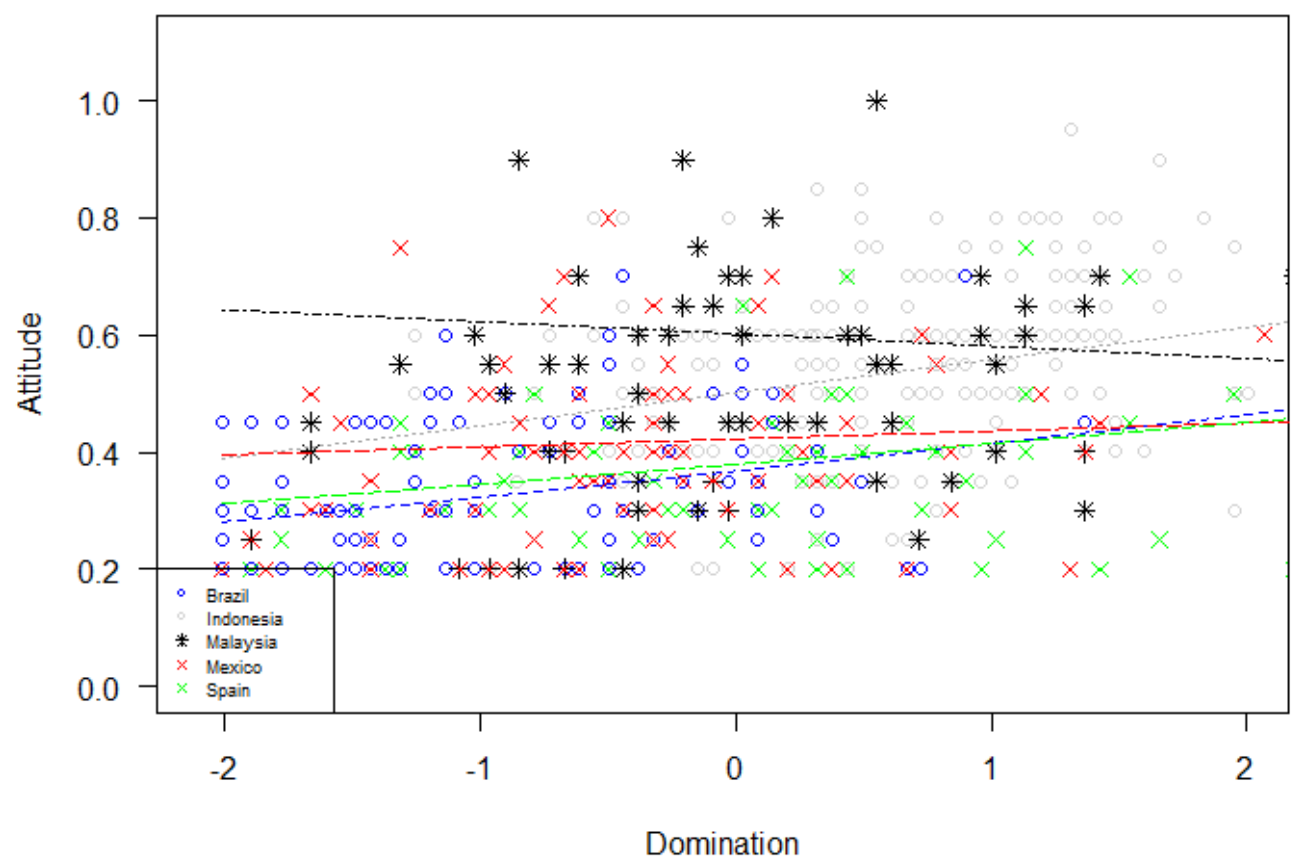

Figure 3. Mean level of participants' attitude toward wildlife (i.e., a low score represents a better attitude), as a function of their country and domination orientations. Circles, crosses, and asterisks represent participants. The dashed lines depict the model (as in model 3, but separately for each country), which has been back-transformed from the log-odds ratio scale and contains standardized controls. 


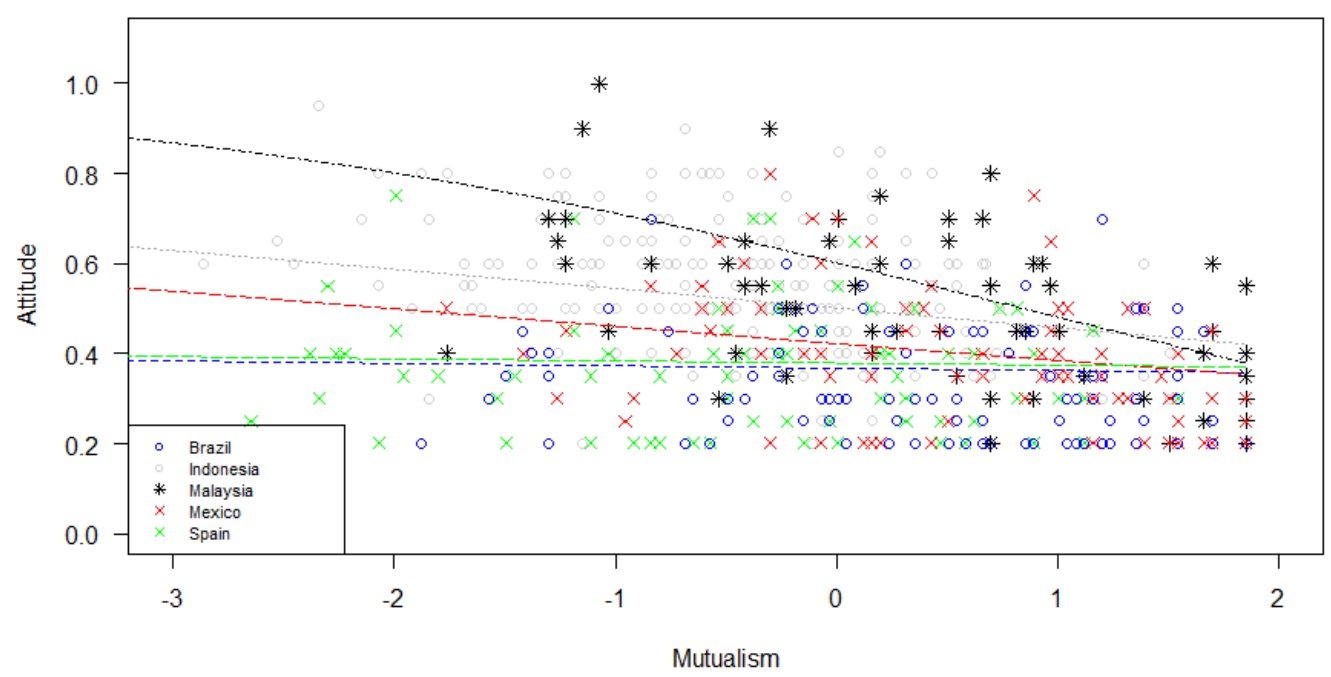

Figure 4. Mean level of participants' attitude toward wildlife (i.e., a low score represents a better attitude), as a function of their country and mutualism orientations. Circles, crosses, and asterisks represent participants. The dashed lines depict the model (as in model 3, but separately for each country), which has been back-transformed from the log-odds ratio scale and contains standardized controls.

\section{Discussion}

In this study, we used a cross-cultural perspective to investigate the link between modernization, anthropomorphism, value orientations (i.e., mutualism or domination) and attitudes towards wildlife. Overall, we found that the link between modernization and anthropomorphism was culturally mediated, with modernization being linked to higher anthropomorphism in some countries (i.e., Indonesia, Malaysia, Spain), but not in others (i.e., Brazil, Mexico). In turn, higher levels of anthropomorphism were linked to higher mutualism and lower domination orientations, and these to a more positive attitude towards wildlife, independently of participants' country. These results suggest that, while the link between modernization and anthropomorphism is culturally mediated, higher anthropomorphism universally predicts higher mutualism orientations and more positive attitudes towards wildlife.

In our study, higher levels of modernization (i.e., higher formal education, income, access to technologies and modern experience with animals) predicted higher anthropomorphism, but this effect was culturally mediated, in line with our first prediction (Table 1). In particular, modernization predicted higher anthropomorphism in participants from Indonesia, Malaysia and Spain, but not in Latin America, having no effect on anthropomorphism in Brazil, and predicting lower levels of anthropomorphism in Mexico. A possible explanation for this difference may be that modernization in Mexico further disrupts traditional cultural practices and beliefs. Indigenous groups living in closer contact with nature may be more likely to attribute human features to animals as a reminiscence of preHispanic religious influence (i.e., nahualism, see [47]). In this case, modernization would have a negative impact on anthropomorphism, leading to the loss of traditional beliefs and ultimately decreasing anthropomorphism. Although our study cannot exactly explain which cultural aspects may be relevant for the emergence of these differences, it clearly shows that the link between modernization and anthropomorphism is culturally mediated and cannot be generalized across countries. Moreover, it suggests that general cultural differences between countries cannot alone explain the different role that modernity plays on anthropomorphism across countries (e.g., Spain and Mexico might be considered to be culturally more similar than Spain and Indonesia, yet the effect of modernization on anthropomorphism is more similar between Spain and Indonesia, than between Spain and Mexico). Therefore, future studies should aim to include more countries, and especially to better disentangle which cultural aspects exactly modulate the link between modernization 
and anthropomorphism, and exactly explain the differences across countries that we have evidenced here.

In line with our predictions (Table 1), anthropomorphism was linked to higher mutualism and lower domination orientations, consistently across countries. Our results show than domination was highest in Asian countries, intermediate in Europe and lowest in Latin America. Furthermore, higher levels of anthropomorphism predicted higher mutualism in all countries, especially in Malaysia. Attributing human characteristics to non-human agents is a psychological mechanism that is deeply rooted in human behaviour, emerging early on during development and likely being universal (see $[36,48,49])$. Therefore, it is not surprising that anthropomorphism was consistently linked to higher mutualism and lower domination orientations across countries, despite some differences in the intensity of these interactions. Indeed, these findings are also consistent with other research in Western and non-Western countries, in which higher anthropomorphism was consistently linked to lower domination and/or higher mutualism [19,30,50,51] (but see [39]). In line with our predictions (Table 1), we also found that a more positive attitude to wildlife was predicted by lower domination and higher mutualism. Overall, our results are consistent with abundant literature showing a link between value orientations and attitudes toward wildlife (e.g., lethal removal of wolves in the USA [11], recreational hunting in Germany [39], and hunting in the Netherlands [10]). In the future, it would be interesting to use empirical procedures to directly assess whether changes in anthropomorphism, orientations and attitude really result in behavioural changes, as human intentions do not always correspond to their practices, and positive attitudes may not necessarily result in more positive behaviours toward wildlife (see [34]).

Finally, our results show a significant effect of several control predictors (see Table 3), largely in line with existing literature. For instance, modern attitudes towards pets and vegetarian/vegan diets were linked to higher anthropomorphism (M1), whereas pet ownership predicted higher mutualism (M2b), as previously shown by other studies [52-55]. Being a male, having idiocentric tendencies and following no vegetarian/vegan diet predicted higher domination (M2a), whereas females, individuals with higher modernization scores and those having more allocentric tendencies also had higher mutualism (M2b), in line with literature ([56-59]; see [60,61] for a review). Finally, older participants were also more likely to have a negative attitude toward wildlife (M3), in line with previous studies showing that older people are for instance more likely to support hunting [10,61-63]. Future studies should better differentiate participants' responses depending on the target species (possibly accounting for their phylogenetic proximity to humans), and the frequency and type of previous direct experience that participants had with it, as attitudes may vary depending on the target species, and across cultures (see [30,64]).

In conclusion, our study shows that the link between modernization and anthropomorphism is not universal, but it varies across countries and is therefore likely to be culturally mediated. In some cultures, modernization might increase anthropomorphism (see Manfredo et al., 2016). In others, however, modernization might have the opposite effect, decreasing anthropomorphism and ultimately deteriorating individuals' attitude towards wildlife. Therefore, it highlights the necessity to incorporate cultural variables to the study of how modernization is linked to anthropomorphism, whereas it also confirms that changes in anthropomorphism are generally associated with changes in value orientations and attitudes toward wildlife across cultures. Clearly, our study must be considered a first preliminary approach to the study of cross-cultural variation in the link between modernization and anthropomorphism. In the future, it would be essential to replicate these findings by including more countries and larger sample sizes, to also account for crucial differences across communities within the same country. Moreover, including larger sample sizes would allow researchers to explicitly assess the role that specific demographic and cultural variables (e.g., religious beliefs) play in these processes, and collect information from more representative samples. Our study, for instance, was severely limited by the fact that participants were mainly recruited among university 
students. Despite these limits, we believe that this study can warn us against the negative impact that modernization might have on human attitude towards wildlife, at least in some cultures, and it is an important first step into the study of cross-cultural variation in the link between modernization and anthropomorphism. Although previous research has shown a link between higher levels of modernization and higher levels of anthropomorphism, this link may not be true across all cultures, so that modernization might actually be linked to lower anthropomorphism in some cultures, with deleterious effects for conservation issues. Therefore, our results call for caution when generalizing findings across countries, and should remind conservation policy makers about the importance of always taking particular cultural contexts into account.

Author Contributions: J.L.G.-M. and F.A. designed the research; J.L.G.-M., R.A.-N., P.I. and S.A.M.S., J.P., R.I.M. and P.O.N. collected data; F.A. and J.L.G.-M. Conducted statistical analysis; F.A. and J.L.G.-M., R.A.-N., P.I., S.A.M.S., J.P., R.I.M., P.O.N., B.M., T.R. and F.A. wrote the paper. All authors have read and agreed to the published version of the manuscript.

Funding: We are grateful to the University of Leipzig and the Max Planck Institute for Evolutionary Anthropology for support during this study, to the Ministerio de Ciencia, Innovación y Universidades that funded Jose Gómez-Melara (FPU 16/02878) during his PhD, and to the FAPESP (14/13237-1) for funding to Patrícia Izar that allowed access to local communities during this research. We acknowledge support from the Max Planck Society for the opportunity to publish Open Access.

Institutional Review Board Statement: The study was conducted according to the guidelines of the Declaration of Helsinki. This research has been approved by the UPD and the Comision de Doctorado del Vicerrectorado de Investigación of the University of Seville.

Informed Consent Statement: Informed consent was obtained from all subjects involved in the study and approved by the UPD of the University of Seville.

Data Availability Statement: Data will be able online after publication.

Acknowledgments: We are grateful to Laura Amores, Iván Periañez, Jose María Sánchez, Virginia Alba, Jose Luis Escacena, Plácido Fernandez-Viagas for their help during data collection. We thank the whole team at CACTUS-lab for their help and generosity adapting our questionnaire to the online version of Ethnoap.

Conflicts of Interest: The authors declare no conflict of interest.

\section{Appendix A}

Participants

We recruited 741 participants across five countries (Brazil, $\mathrm{N}=156$, Indonesia, $\mathrm{N}=248$, Malaysia, $\mathrm{N}=118$, Mexico, $\mathrm{N}=111$, Spain, $\mathrm{N}=108$ ). Participants answering this question described themselves as females $(\mathrm{N}=394)$ or males $(\mathrm{N}=240)$, with no representation of other genders. Participants' age ranged from 18 to 79 (mean $=28.76)$. Below, we report more detailed information for each country.

Table A1. Gender distribution by country.

\begin{tabular}{cccccc}
\hline & Brazil & Indonesia & Malaysia & Mexico & Spain \\
\hline Female & 79 & 146 & 59 & 60 & 50 \\
Male & 44 & 101 & 18 & 26 & 51 \\
N/A & 33 & 1 & 41 & 25 & 7 \\
\hline
\end{tabular}


Table A2. Age distribution by country *

\begin{tabular}{cccccc}
\hline & Brazil & Indonesia & Malaysia & Mexico & Spain \\
\hline$<20$ & 3 & 137 & 1 & 0 & 70 \\
$20-29$ & 29 & 60 & 75 & 26 & 25 \\
$30-39$ & 24 & 30 & 0 & 39 & 0 \\
$40-49$ & 16 & 15 & 1 & 9 & 0 \\
$50-59$ & 30 & 6 & 0 & 10 & 3 \\
$60-69$ & 12 & 0 & 0 & 2 & 3 \\
$70-79$ & 7 & 0 & 0 & 1 & 0 \\
\hline
\end{tabular}

* Age has been grouped into categories in this Table for clarity, but was used as a continuous variable in our models.

Table A3. Education level distribution by country.

\begin{tabular}{cccccc}
\hline & Brazil & Indonesia & Malaysia & Mexico & Spain \\
\hline No education & 0 & 1 & 0 & 0 & 0 \\
Primary school & 0 & 15 & 0 & 0 & 0 \\
High school & 39 & 218 & 33 & 14 & 88 \\
Bachelor & 13 & 14 & 41 & 34 & 7 \\
Postgraduate & 69 & 0 & 1 & 38 & 2 \\
N/A & 35 & 0 & 43 & 25 & 11 \\
\hline
\end{tabular}

Table A4. Dietary preference distribution by country.

\begin{tabular}{cccccc}
\hline & Brazil & Indonesia & Malaysia & Mexico & Spain \\
\hline Omnivorous & 93 & 179 & 42 & 77 & 92 \\
Vegan or vegetarian & 27 & 63 & 29 & 8 & 8 \\
N/A & 36 & 6 & 47 & 26 & 8 \\
\hline
\end{tabular}

\section{Appendix B}

\section{Questionnaire}

The questionnaire contained several questions that allowed us to build our test predictors and dependent variables. First, we assessed participants' tendency to make anthropomorphic attributions (i.e., anthropomorphism), by assigning participants a score that varied between 0 (i.e., minimum level of anthropomorphism) and 1 (i.e., maximum level of anthropomorphism). To calculate this score, we used the 5 statements by Manfredo et al. [18] to increase comparability, which included sentences like "Animals have free will". Participants could rate these statements on a 5-point agreement scale, from "strongly disagree" (1) to "strongly agree" (5). The anthropomorphism score was then obtained by summing all these points and dividing the sum by the maximum value that the participant could obtain (i.e., if the participant provided no answer to one of the 5 statements, the maximum value that could be obtained was 20). Second, we assessed participants' domination orientation, giving participants a score from 0 (i.e., minimum domination level) to 1 (i.e., maximum domination level). To calculate it, we scored individual responses to the 10 statements used by Manfredo et al. [18], including 5 domination statements such as "Humans should manage fish and wildlife populations so that humans benefit" and 5 hunting statements such as "People who want to hunt animals should be provided the opportunity to do so". As above, participants could rate these statements on a 5-point agreement scale. We summed the points obtained for each 5-statement group and divided the sums by the maximum value a participant could obtain (as above). The mean of these two values was the participants' domination orientation. Similarly, we assessed participants' mutualism orientation, by assigning them a score from 0 (i.e., minimum mutualism level) to 1 (i.e., maximum mutualism level). As above, it was based on 5 mutualism statements like "We should strive for a world where there is an abundance of fish and wildlife for hunting and fishing" and 5 hunting statements like "Hunting is cruel and inhumane to the animals" [19]. Participants could rate these statements on a 5-point agreement scale (as above). 
We summed all points for each 5-statement group, divided the sums by the maximum value participants could obtain, and used the mean of these two values as the participants' mutualism orientation.

Finally, we assessed other possible factors that might predict and/or modulate participants', anthropomorphism, domination and mutualism orientations towards wildlife. First, we assessed attitude towards wildlife, assigning individuals a score from 0 (i.e., negative attitude towards wildlife) to 1 (i.e., positive attitude towards wildlife). We calculated this score based on participants' response to 4 statements about human-wildlife conflict, like "Animals are a pest messing up the place where I live" (see Karimullah et al., in revision), to be rated on a 5-point agreement scale (as above). Participants' attitude was calculated by summing all the points obtained by each individual and dividing the sum by the maximum value that the participant could have obtained (as above). Second, we assessed participants' degree of modernization by using 8 questions and scoring the following four aspects of modernization: (i) individual income (i.e., assigning 1 to the individual declaring the highest income in each country and proportionally assigning a value to the other participants, so that income in each country varied from 0 to 1 ); (ii) level of formal education (i.e., with individuals who did not conclude primary school scoring 0.2 , those who concluded primary school, secondary school or university $0.4,0.6$ or 0.8 , respectively, and those having higher degrees scoring 1); (iii) modern experience with animals (i.e., with individuals scoring up to 1 , depending on the proportion of positive answers given to 4 questions, like "Have you ever visited a zoo, a natural reserve or a similar place?"); and (iv) access to technologies (i.e., with individuals scoring up to 1 on daily use of technologies and social media, based on three questions assessing their use of social networks, hours spent watching TV and using internet). Finally, we summed the four scores (i.e., income, formal education, modern experience with animals and access to technologies), and divided the sum for the maximum score that participants could have obtained (i.e., if they provided no answer to the questions on one of the four aspects of modernization, the maximum score they could have obtained was three, so that modernization could vary between 0 (i.e., minimum level of modernization) and 1 (i.e., maximum level of modernization). Third, we assessed pet-ownership dynamics (i.e., ownership and affection to pets). We assigned participants a score from 0 (i.e., minimum level) to 1 (i.e., maximum level), based on their response to three yes-or-no questions such as "Do you own any animal?" The score was obtained by summing all the points obtained and dividing the sum for the maximum value that could be obtained (as above). Fourth, we assessed participants' sociality (i.e., frequency of social interactions) by assigning them a score between 0 (i.e., low sociality) and 9 (i.e., high sociality). We used a set of nine open questions such as "How many times do you hang out in an average week?" For each question, we then assigned 1 to the individual declaring the highest number, and proportionally assigned a value to the other participants so that these values varied from 0 to 1 . For each participant, we then calculated the sociality score as the mean of her/his nine answers. Fifth, we assessed participants' idiocentric and allocentric tendencies (i.e., idio-allocentrism index) by giving participants a number from 0 (i.e., high allocentrism) to 1 (i.e., high idiocentrism). For this purpose, we used a validated questionnaire containing 16 statements (as in [41,42]), such as "I would rather depend on myself than others". Participants could rate these statements on a 5-point agreement scale (as above). Idiocentric and allocentric tendencies were then assessed by summing all the points obtained and dividing them by the maximum value that they could have obtained (as above). Finally, we collected some demographic information with open questions including participants' age, gender and dietary preferences (i.e., whether they were vegetarian or vegan).

\section{References}

1. Dirzo, R.; Young, H.S.; Galetti, M.; Ceballos, G.; Isaac, N.J.B.; Collen, B. Defaunation in the Anthropocene. Science 2014, 345, 401-406. [CrossRef] [PubMed]

2. Goldberg, T.L. Forest Fragmentation as Cause of Bacterial Transmission among Nonhuman Primates, Humans, and Livestock, Uganda. Emerg. Infect. Dis. 2008, 14, 1375-1382. [CrossRef] 
3. Naughton-Treves, L. Predicting patterns of crop damage by wildlife around Kibale National Park, Uganda. Conserv. Biol. 1998, 12, 156-168. [CrossRef]

4. Woodroffe, R.; Thirgood, S.; Rabinowitz, A. People and Wildlife, Conflict or Co-Existence? Cambridge University Press: Cambridge, UK, 2005.

5. Reidinger, R.; Miller, J. Wildlife Damage Management: Prevention, Problem Solving and Conflict Resolution; Johns Hopkins University Press: Baltimore, MD, USA, 2013.

6. Stern, M.J.; Powell, R.B.; Hill, D. Environmental education program evaluation in the new millennium: What do we measure and what have we learned? Environ. Educ. Res. 2014, 20, 581-611. [CrossRef]

7. Nyhus, P.J. Human-Wildlife Conflict and Coexistence. Annu. Rev. Environ. Resour. 2016, 41, 143-171. [CrossRef]

8. Bruskotter, J.T.; Vucetich, J.A.; Dietsch, A.; Slagle, K.M.; Brooks, J.S.; Nelson, M.P. Conservationists' moral obligations toward wildlife: Values and identity promote conservation conflict. Biol. Conserv. 2019, 240, 108296. [CrossRef]

9. Manfredo, M.J.; Teel, T.L.; Sullivan, L.; Dietsch, A.M. Values, trust, and cultural backlash in conservation governance: The case of wildlife management in the United States. Biol. Conserv. 2017, 214, 303-311. [CrossRef]

10. Vaske, J.J.; Jacobs, M.H.; Sijtsma, M.T.J. Wildlife value orientations and demographics in The Netherlands. Eur. J. Wildl. Res. 2011, 57, 1179-1187. [CrossRef]

11. Manfredo, M.J.; Teel, T.L.; Don Carlos, A.W.; Sullivan, L.; Bright, A.D.; Dietsch, A.M.; Bruskotter, J.; Fulton, D. The changing sociocultural context of wildlife conservation. Conserv. Biol. 2020, 34, 1549-1559. [CrossRef]

12. Manfredo, M.J.; Teel, T.L.; Henry, K.L. Linking Society and Environment: A Multilevel Model of Shifting Wildlife Value Orientations in the Western United States. Soc. Sci. Q. 2009, 90, 407-427. [CrossRef]

13. Maarten, J.; Vaske, J.; Teel, T.; Manfredo, M.J. Human dimensions of wildlife. In Environmental Psychology: An introduction; Steg, L., Van den Berg, A., de Groot, J., Eds.; BPS Backwell; John Wiley \& Sons: Hoboken, NJ, USA, 2007; pp. 78-86.

14. Teel, T.; Manfredo, M.J. Understanding the diversity of public interests in wildlife conservation. Conserv. Biol. 2010, 24, 128-139. [CrossRef]

15. Teel, T.; Manfredo, M.J.; Miller, H.M. The Need and Theoretical Basis for Exploring Wildlife Value Orientations Cross-Culturally. Hum. Dimens. Wildl. An. Int. J. 2007, 12, 297-305. [CrossRef]

16. Schwartz, B. The Paradox of Choice: Why More Is Less; Eco: New York, NY, USA, 2004.

17. Inglehart, R.; Welzel, C. Modernization, Cultural Change, and Democracy: The Human Development Sequence; Cambridge University Press: New York, NY, USA, 2005.

18. Julius, H.; Beetz, A.; Kotrschal, K.; Turner, D.; Uvnäs-Moberg, K. Attachment to Pets: An Integrative View of Human-Animal Relationships with Implications for Therapeutic Practice; Hogrefe Publishing: Göttingen, Germany, 2012.

19. Manfredo, M.J.; Urquiza-Haas, E.G.; Don Carlos, A.W.; Bruskotter, J.T.; Dietsch, A.M. How anthropomorphism is changing the social context of modern wildlife conservation. Biol. Conserv. 2019, 241, 108297. [CrossRef]

20. Bruskotter, J.T.; Vucetich, J.A.; Manfredo, M.J.; Karns, G.R.; Wolf, C.; Ard, K.; Carter, N.H.; López-Bao, J.V.; Chapron, G.; Gehrt, S.D.; et al. Modernization, Risk, and Conservation of the World's Largest Carnivores. Bioscience 2017, 67, 646-655. [CrossRef]

21. Vaske, J.J.; Donnelly, M.P. A value-attitude-behavior model predicting wildland preservation voting intentions. Soc. Nat. Resour. 1999, 12, 523-537. [CrossRef]

22. Driscoll, J. Attitudes toward Animals: Species Ratings Janis. Soc. Anim. 1993, 3, 139-149. [CrossRef]

23. Eddy, T.J.; Gallup, G.G.; Povinelli, D.J. Attribution of Cognitive States to Animals: Anthropomorphism in Comparative Perspective. J. Soc. Issues 1993, 49, 87-101. [CrossRef]

24. Harrison, M.A.; Hall, A.E. Anthropomorphism, empathy, and perceived communicative ability vary with phylogenetic relatedness to humans. J. Soc. Evol. Cult. Psychol. 2010, 4, 34-48. [CrossRef]

25. Martín-Forés, I.; Martín-López, B.; Montes, C. Anthropomorphic Factors Influencing Spanish Conservation Policies of Vertebrates. Int. J. Biodivers. 2013, 2013, 142670. [CrossRef]

26. Martín-López, B.; Montes, C.; Benayas, J. The non-economic motives behind the willingness to pay for biodiversity conservation. Biol. Conserv. 2007, 139, 67-82. [CrossRef]

27. Williams, M.; Whitmarsh, L.; Chriost, D. The association between anthropomorphism of nature and pro-environmental variables: A systematic review. Biol. Conserv. 2021, 255, 109022. [CrossRef]

28. Díaz, E.M. Animal Humanness, Animal Use, and Intention to Become Ethical Vegetarian or Ethical Vegan. Anthrozoos 2016, 29, 263-282. [CrossRef]

29. Wang, F.; Basso, F. "Animals are friends, not food": Anthropomorphism leads to less favorable attitudes toward meat consumption by inducing feelings of anticipatory guilt. Appetite 2019, 138, 153-173. [CrossRef] [PubMed]

30. Higgs, M.J.; Bipin, S.; Cassaday, H.J. Man's best friends: Attitudes towards the use of different kinds of animal depend on belief in different species' mental capacities and purpose of use. R. Soc. Open Sci. 2020, 7, 109022. [CrossRef]

31. Apostol, L.; Rebega, O.L.; Miclea, M. Psychological and Socio-demographic Predictors of Attitudes toward Animals. Procedia-Soc. Behav. Sci. 2013, 78, 521-525. [CrossRef]

32. Tam, K.P.; Lee, S.L.; Chao, M.M. Saving Mr. Nature: Anthropomorphism enhances connectedness to and protectiveness toward nature. J. Exp. Soc. Psychol. 2013, 49, 514-521. [CrossRef]

33. Tam, K.P. Dispositional empathy with nature. J. Environ. Psychol. 2013, 35, 92-104. [CrossRef] 
34. Tam, K.P. Anthropomorphism of nature, environmental guilt, and pro-environmental behavior. Sustainability 2019, 11, 5430. [CrossRef]

35. Chin, M.G.; Sims, V.K.; Clark, B.; Lopez, G.R. Measuring Individual Differences in Anthropomorphism toward Machines and Animals. Proc. Hum. Factors Ergon. Soc. Annu. Meet. 2004, 48, 1252-1255. [CrossRef]

36. Severson, R.L.; Lemm, K.M. Kids See Human Too: Adapting an Individual Differences Measure of Anthropomorphism for a Child Sample. J. Cogn. Dev. 2016, 17, 122-141. [CrossRef]

37. Letheren, K.; Kuhn, K.A.L.; Lings, I.; Pope, N.K.L. Individual difference factors related to anthropomorphic tendency. Eur. J. Mark. 2016, 50, 973-1002. [CrossRef]

38. Wilkins, A.M.; McCrae, L.S.; McBride, E.A. Factors affecting the human attribution of emotions toward animals. Anthrozoos 2015, 28, 357-369. [CrossRef]

39. Riepe, C.; Arlinghaus, R. Explaining Anti-Angling Sentiments in the General Population of Germany: An Application of the Cognitive Hierarchy Model. Hum. Dimens. Wildl. 2014, 19, 371-390. [CrossRef]

40. Hawkins, R.D.; Williams, J.M. Children's attitudes towards animal cruelty: Exploration of predictors and socio-demographic variations. Psychol. Crime Law 2020, 26, 226-247. [CrossRef]

41. Triandis, H.C.; Gelfand, M.J. Converging Measurement of Horizontal and Vertical Individualism and Collectivism. J. Pers. Soc. Psychol. 1998, 74, 118-128. [CrossRef]

42. Triandis, H.C.; Leung, K.; Villareal, M.J.; Clack, F.I. Allocentric versus idiocentric tendencies: Convergent and discriminant validation. J. Res. Pers. 1985, 19, 395-415. [CrossRef]

43. Baayen, R.H.; Davidson, D.J.; Bates, D.M. Mixed-effects modeling with crossed random effects for subjects and items. J. Mem. Lang. 2008, 59, 390-412. [CrossRef]

44. R-CoreTeam. R: A Language and Environment for Statistical Computing; R Foundation for Statistical Computing: Vienna, Austria, 2020.

45. Brooks, M.E.; Kristensen, K.; van Benthem, K.J.; Magnusson, A.; Berg, C.W.; Nielsen, A.; Skaug, H.J.; Mächler, M.; Bolker, B.M. glmmTMB balances speed and flexibility among packages for zero-inflated generalized linear mixed modeling. $R \mathrm{~J} . \mathbf{2 0 1 7}, 9$, 378-400. [CrossRef]

46. Smithson, M.; Verkuilen, J. A better lemon squeezer? Maximum-likelihood regression with beta-distributed dependent variables. Psychol. Methods 2006, 11, 54-71. [CrossRef]

47. Martínez-González, R. El Nahualismo; Universidad Nacional Autónoma de México: Ciudad de Mexico, Mexico, 2011.

48. Epley, N.; Waytz, A.; Cacioppo, J. On Seeing Human: A Three-Factor Theory of Anthropomorphism. Psychol. Rev. 2007, 114, 864-886. [CrossRef]

49. Nyhof, M.A.; Johnson, C.N. Is God just a big person? Children's conceptions of God across cultures and religious traditions. Br. J. Dev. Psychol. 2017, 35, 60-75. [CrossRef] [PubMed]

50. Abidin, Z.A.Z.; Jacobs, M.H. The Applicability of Wildlife Value Orientations Scales to a Muslim Student Sample in Malaysia. Hum. Dimens. Wildl. 2016, 21, 555-566. [CrossRef]

51. Abidin, Z.A.Z.; Jacobs, M. Relationships between valence towards wildlife and wildlife value orientations. J. Nat. Conserv. 2019, 49, 63-68. [CrossRef]

52. Bjerke, T.; Østdahl, T.; Kleiven, J. Attitudes and activities related to urban wildlife: Pet owners and non-owners. Anthrozoos 2003, 16, 252-262. [CrossRef]

53. Prokop, P.; Tunnicliffe, S.D. Effects of having pets at home on children's attitudes toward popular and unpopular animals. Anthrozoos 2010, 23, 21-35. [CrossRef]

54. Rothgerber, H.; Mican, F. Childhood pet ownership, attachment to pets, and subsequent meat avoidance. The mediating role of empathy toward animals. Appetite 2014, 79, 11-17. [CrossRef]

55. Shuttlewood, C.Z.; Greenwell, P.J.; Montrose, V.T. Pet Ownership, Attitude toward Pets, and Support for Wildlife Management Strategies. Hum. Dimens. Wildl. 2016, 21, 180-188. [CrossRef]

56. Dougherty, E.M.; Fulton, D.C.; Anderson, D.H. The influence of gender on the relationship between wildlife value orientations, beliefs, and the acceptability of lethal deer control in Cuyahoga Valley National Park. Soc. Nat. Resour. 2003, 16, 603-623. [CrossRef]

57. Heleski, C.R.; Mertig, A.G.; Zanella, A.J. Stakeholder attitudes toward farm animal welfare. Anthrozoos 2006, 19, $290-307$. [CrossRef]

58. Zinn, H.C.; Pierce, C.L. Values, gender, and concern about potentially dangerous wildlife. Environ. Behav. 2002, 34, 239-256. [CrossRef]

59. Randler, C.; Ballouard, J.M.; Bonnet, X.; Chandrakar, P.; Pati, A.K.; Medina-Jerez, W.; Pande, B.; Sahu, S. Attitudes toward Animal Welfare Among Adolescents from Colombia, France, Germany, and India. Anthrozoos 2021, 34, 359-374. [CrossRef]

60. Herzog, H.A. Gender differences in human-animal interactions: A review. Anthrozoos 2007, 20, 7-21. [CrossRef]

61. Ormandy, E.H.; Schuppli, C.A. Public attitudes toward animal research: A review. Animals 2014, 4, 391-408. [CrossRef] [PubMed]

62. Donnelly, M.P.; Vaske, J.J. Predicting attitudes toward a proposed moose hunt. Soc. Nat. Resour. 1995, 8, 307-319. [CrossRef]

63. Gamborg, C.; Jensen, F.S. Attitudes towards recreational hunting: A quantitative survey of the general public in Denmark. J. Outdoor Recreat. Tour. 2017, 17, 20-28. [CrossRef]

64. Urquiza-Haas, E.G.; Kotrschal, K. The mind behind anthropomorphic thinking: Attribution of mental states to other species. Anim. Behav. 2015, 109, 167-176. [CrossRef] 